\title{
Adaptive Router Promotion in Ad-Hoc Networks
}

\author{
Kensuke Tanaka, Noriko Matsumoto, and Norihiko Yoshida* \\ Department of Information and Computer Sciences \\ Saitama University, Saitama 338-8570, Japan \\ * yoshida@ics.saitama-u.ac.jp
}

\begin{abstract}
In ad-hoc networks, routing is one of the most important issues, and various protocols are proposed. However, as situations and topologies of an ad-hoc network are various and dynamic, it is difficult for a single fixed protocol to perform well for all occasions. Therefore, some dynamic and adaptive mechanism in routing protocols is necessary. In this paper, we propose an adaptive routing system for ad-hoc networks. This system begins in the same manner as a reactive protocol, and when the network situation gets unsuitable for the protocol, the system changes its manner of routing with a router-node, or a pseudo cluster-head in Cluster-based Routing, which emerges autonomously at the place of "hot spots" in the network. This paper presents its principle, design, and some preliminary experiment results.
\end{abstract}

Keywords: Ad-hoc Network, Cluster-based Routing, Reactive Routing, Adaptive Network, Network Traffic Reduction.

\section{Introduction}

Ad-hoc networks are autonomously constructed from end-user nodes without any particular network equipments, and are expected to be used in various occasions. Among several issues to be addressed, routing is one of the most important and difficult subjects.

In a basic ad-hoc network, there is no node like a router that manages composition of the whole network and performs routing. Each node must obtain routing information by itself, and determines a route according to the information. Various routing protocols for ad-hoc networks have already been devised and proposed. However, because situation and topology of an ad-hoc network changes dynamically, it is almost impossible to determine which protocol is the optimal in advance. The optimal protocol must change according to the movement of nodes, the variation of communication frequencies, etc.

To solve this problem, we propose an adaptive routing system which changes the manner of routing to be suitable for the network situation dynamically. The purpose of our system is to alleviate or prevent "flooding", in which every node broadcasts and propagates search packets, by promoting a node at a "hot 


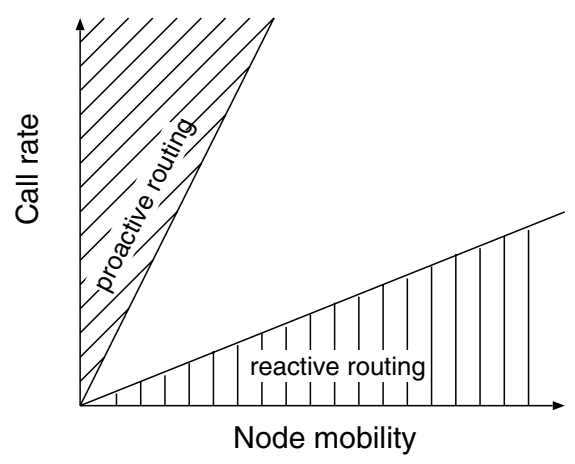

Fig. 1. Ad-Hoc Network Design Space

spot" in the network to a router-like node, or a pseudo cluster head in Clusterbased Routing, which every other node around can ask it for routing information instead of flooding.

In the following, Section 2 summarizes routing protocols for ad-hoc networks. Basic protocols, proactive ones and reactive ones, are first presented, and then some improvement including hybrid routing and Cluster-based Routing are described. Our solution proposal, an adaptive routing system, is presented in Section 3, and in 4 in details. Section 5 describes an experiment result for evaluation, and Section 6 considers some comparisons. The last section contains some concluding remarks.

\section{Routing in Ad-Hoc Networks}

\subsection{Basic Protocols}

In basic ad-hoc networks, each node must obtain necessary routing information by itself. Consequently, various routing protocols have been developed, which are primarily categorized as proactive routing protocols (OLSR [1], TBRPF [2], etc.) and reactive routing protocols (AODV [3, DSR 4, etc.)

As is well known, each of the proactive and reactive protocols performs well only in a limited situation regarding operational conditions and network configurations that should be covered by ad-hoc networks. Fig. 1 shows the design space of ad-hoc networks with node mobility and call rate as the two dimensions, and the approximate regions where each of these two protocols performs well [5].

If the node mobility is higher, the possibility that the route expires immediately is also higher. Therefore, the reactive protocols which builds routes only when needed are more effective than the proactive ones. On the other hand, if call rate is higher, the proactive protocols are more effective than the reactive ones in which a node must look for a route whenever it calls another. The white region in the center of Fig. 1 corresponds to network situations that neither of two basic protocols performs well. 
(R)

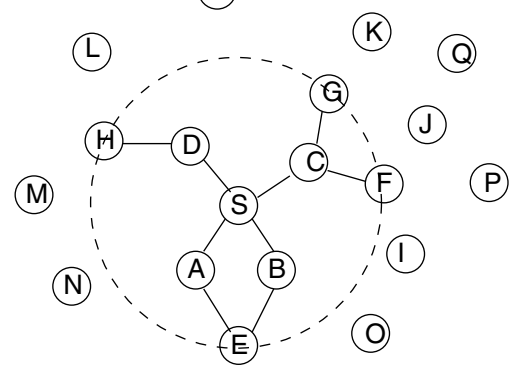

Fig. 2. Zone Routing with 2-hops-radius Zone

\section{$2.2 \quad$ Hybrid Routing}

As described above, each basic protocol is suited for a different region of the adhoc network design space. In hybrid routing, each node uses different protocols by combining them into a single framework. One of the most famous hybrid routing is "Zone Routing" 6].

In Zone Routing, a proactive protocol operates within a local area which we refer to as a routing zone (intra-zone routing), and a reactive protocol (interzone routing) operates outside of that. A proactive routing protocol provides a detailed and fresh view of each node's surrounding local topology, and finds a route to distant nodes reactively to reduce the overhead of route maintenance.

Fig. 2 illustrates the routing zone concept with a 2-hops-radius routing zone. The routing zone described by a dashed circle belongs to node $S$, and nodes from $A$ to $H$ are members of $S$ 's routing zone. Note that each node maintains its own routing zone, and the zones of neighbor nodes overlap.

\subsection{Cluster-Based Routing}

Cluster-based Routing, or Clustering, is the technique of dividing the nodes in the network into clusters according to a certain distributed manner 78 .

A cluster head is elected for each cluster to maintain cluster membership information and member routing information. Cluster member nodes do not have routing information, and ask the cluster head whenever needed. Inter-cluster routes are discovered dynamically using the cluster membership information kept at each cluster head (Fig. 33).

\section{Adaptive Routing}

The previous section describes some protocols aiming at covering the situation that neither a pure reactive nor proactive type can work well. All the nodes keep routing information in the proactive protocols, while no node keeps routing information in the reactive ones. In this respect, These two types of the protocols stay at the opposite extremes. This fact is shown also in the above-mentioned 


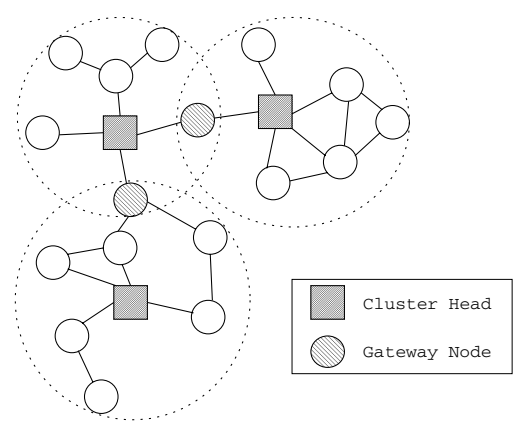

Fig. 3. Cluster-based Routing

Fig. 1 that the regions which these two categories cover two extremes of the ad-hoc network space. Therefore, it can be said that a protocol which performs well in the region between two extremes in an adaptive manner is desired for efficient maintenance of routing information.

Our protocol uses some (but not all) nodes to maintain routing information as shown in Fig. 4. These nodes should be placed where they are the most effective and when they are required. Namely, each node starts in the same manner as in reactive protocols where no node has routing information, and accumulate routing information when transferring routing packets.

When the amount of routing information grows, it implies that the node is at a "crossroad" or a "hot spot" in the network, and neighboring nodes get benefits (from routing standpoint of view) from the node. Therefore, it should be better to make the node as "coordinator" or "router", and make its neighbors into its cluster, so as to prevent flooding of route request packets.

In this manner, this network system transforms its way of routing from reactive to pseudo cluster-based dynamically and adaptively as shown in Fig. 5 .

In this system, the number of the router-nodes and their locations in the network are determined adaptively according to the network traffic in a fully decentralized manner, and clusters emerge dynamically, unlike the above mentioned
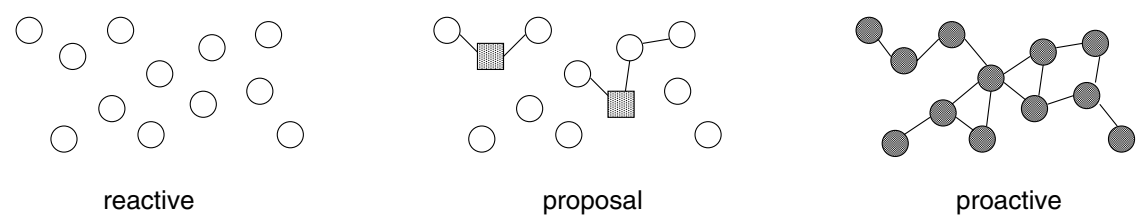

Node

Router-Node

* The depth of color shows the amount of routing information

Fig. 4. Axis of Routing Protocols 
Node S search routing information to Node D
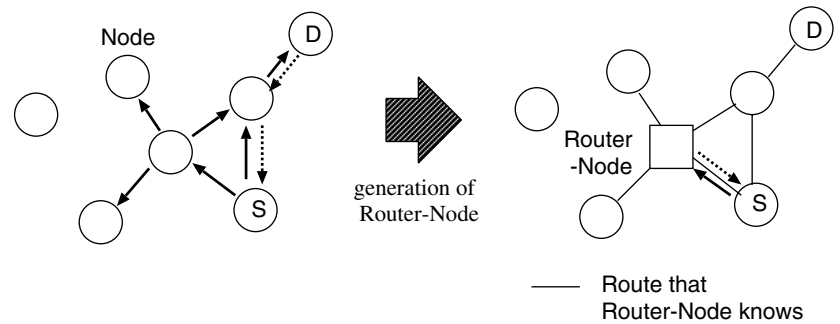

Fig. 5. Emergence of Router-Node

original cluster-based routing in which clusters are pre-defined and fixed in a static manner.

\section{Design Details}

\subsection{The Initial State}

The system performs routing using the reactive AODV protocol in the initial state. Each node sends HELLO packets periodically to confirm connectivity with neighbor nodes. RREQ (Route Request) packets are flooded to find routing information, and if the destination node or an intermediate node which caches routing information to the destination receives RREQ packet, it answers RREP (Route Reply) packet, and the route is built between two nodes.

\subsection{Cache of Routing Information}

Each node caches routing information. If communication frequency goes up, and many routes are used within a short period, the amount of these caches also increases. Each node approximates the network traffic by this amount of its cache, and if this exceeds a certain pre-defined threshold, this fact implies that the node is at a place with high call rate, or a "hot spot." Then the node promotes itself to a router-node. Note that every node has an option whether it can be promoted or not, according to its connectivity and capacity for example.

\subsection{Promotion to Router-Node}

Fig. 6]illustrates the process how the node promotes itself to a router-node, and how neighbor nodes are made to ask routing information to the router-node directly so as to prevent the "flooding" cost of finding routing information.

Suppose that the node $C$ promotes itself to a router-node. $C$ notifies the fact to its neighbor nodes using the "router notification" packet. Then, $C$ collects routing information from its neighbor nodes. A neighbor node $E$ has routing 


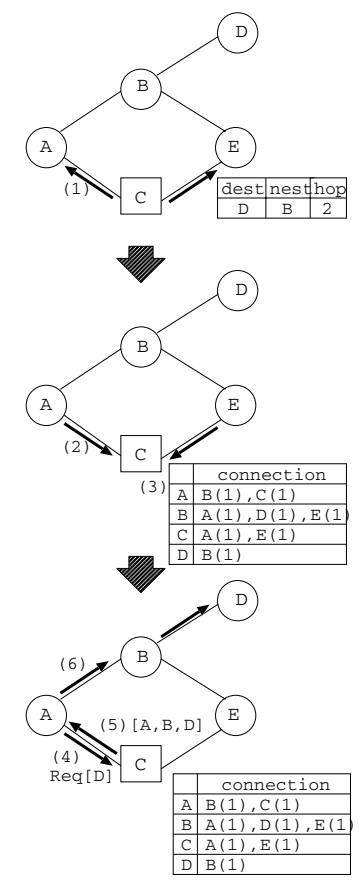

(1) send "router notification" packet to neighbor nodes

(Router-node)

(2) make "topology information" packet from information on the routing table etc. and send it to router-node

(Neighbor nodes)

(3) make topology table from information on the recived "topology information" packet (Router-node)

(4) ask the routing information with sending "shortest route request" packet

(Neighbor nodes)

(5) send "shortest route reply" packet in which the route (sorce route) created from the topology table is stored

(Router-node)

(6) send data packet with sorce route (Neighbor nodes)

Fig. 6. Promotion to Router-node

information such as "to node $D$, next hop is $B$, and hop count is 2 ". Therefore, $E$ composes this information into a "topology information" packet, and sends it to the router-node $C$. $C$ also receives a similar packet from the node $A$. The routernode constructs a network topology table from the collected routing information which is specific to each original node.

When a route request arrives to the router-node $C$, it composes routing information dynamically from this topology table using Dijkstra's shortest-path algorithm. For example, when node $A$ asks $C$ a route to the node $D, C$ replies the shortest route from $A$ to $D$ as $[\mathrm{A}, \mathrm{B}, \mathrm{D}]$.

\subsection{Search for Routing Information}

Each node tries to get routing information in the following order: (1) check whether it has routing information, (2) ask to a known router-node, or (3) flood RREQ packet. The router-node does (1), and then (3); a "plain" node (who does not know any router-node) does (1), and then (3); and a neighbor of the routernode does (1), and then (2). Note that when receiving a RREQ packet from another node, each node does not carry out flooding immediately, but follows the above steps.

A neighbor node which receives a RREQ from a plain node notifies the existence of the router-node to the plain node along with a reply. The plain node 
becomes a new "neighbor" node, and the router information is propagated in this manner.

\subsection{Sending and Forwarding}

In AODV, routing is done in a "hop-by-hop" manner. Each intermediate node has its own routing information from itself to any known destinations. On the other hand, in router-based routing, routing information is provided from the router-node. This is a "source route" that indicates an entire route from the source to the destination. Therefore, our system implements a switching mechanism between the two types of routing. A packet header contains a special flag, and nodes perform appropriate routing based on this flag. This is an application of Active Networks [9].

\subsection{Update of Routing Information}

The routing information in the router-node does not expire unless it is reported obsolete. If the router-node itself or any of its neighbors finds a change in network connectivity, the routing information is updated. Any new routes will possibly be reported to the router-node as well.

\subsection{Demotion of Router-Nodes}

A router-node monitors accesses to itself, and when the access rate decreases under a certain threshold, it demotes itself to a plain node. It still keeps all the routing information, and replies when asked just like the (volatile) cache in a plain node. Its neighbors stop asking routing information to it directly, and go back to use the reactive protocol. The threshold for demotion is set much lower compared to the promotion threshold so as to prevent racing (or thrashing).

\section{Simulation and Experiments}

We have implemented a simulator of our protocol system for design verification and preliminary performance evaluation. It is implemented in Java. The schematic outline of the node implementation is shown in Fig. 7 Each node has two modes of operations: "Normal Agent" and "Routing Agent." The former implements protocols for the plain node operations, and the latter for the router-node operations.

Due to the limit of the space, here we present just one result out of experiments performed on the simulator. Some parameters for the experiment are: the number of nodes is 50, the number of node connection is between 1 to 4 randomly, and the threshold of the cache amount for promoting to a router-node is 15 . The simulator generates request packets from a randomly-chosen source to a randomly-chosen destination.

Fig. 8 shows transition of the total amount of packets in the network. The solid line is of our system, while the dotted line is of a typical reactive protocol 


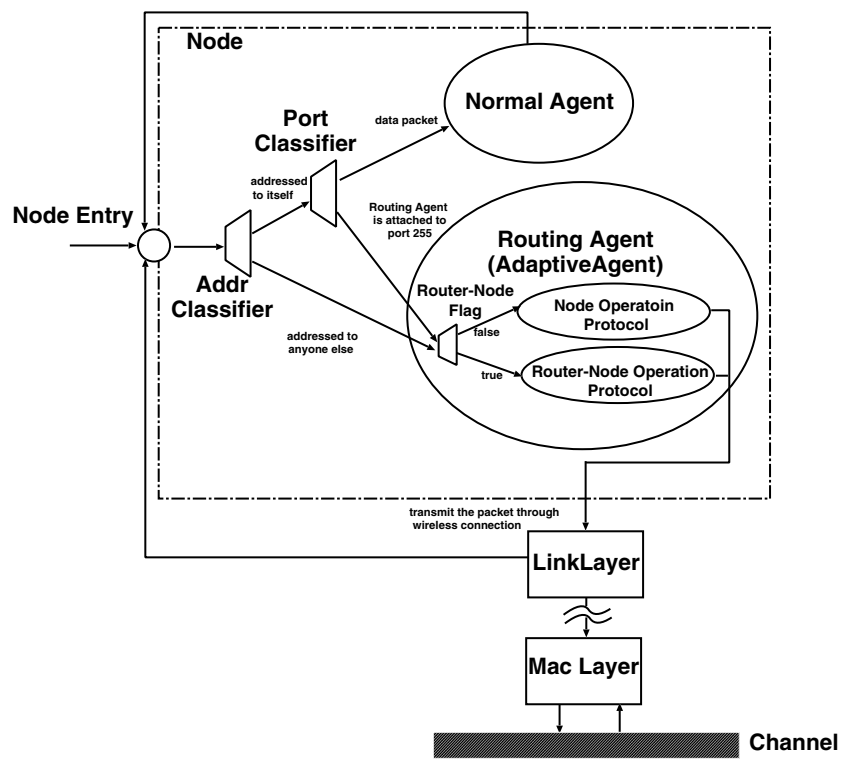

Fig. 7. Outline of Node Implementation

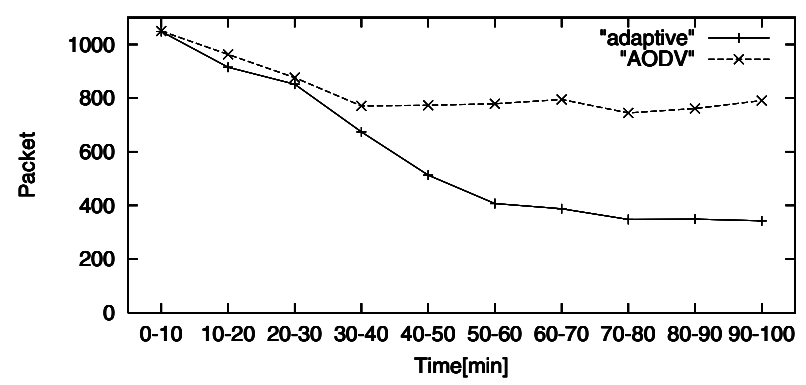

Fig. 8. Experiment Result

AODV. The amount of packets, or network traffic, in our system drastically reduces from the moment of "20-30 (virtual) minute", while the traffic in AODV stays high. Some router-nodes emerge at the same moment as "20-30 minute," so the reduction of traffic is considered brought by the router-nodes. We also observed that the number of emerging route nodes is 2 to 4 , which is sufficiently fewer than the number of all nodes (50).

\section{Considerations}

Our system is considered to have some advantages. First, we compare it with the two basic protocols (reactive and proactive ones). In our system, routing information is managed only on router-nodes. This reduces managing overhead compared to the proactive protocols. On the other hand, neighbor nodes of a 
router-node ask routing information only to the router-node directly instead of flooding RREQ packets. This reduces network traffic significantly compared to the reactive protocols. These mean that, from the route maintenance and network traffic standpoints of view, our systems is positioned somewhere between the proactive protocols and the reactive ones, and expected to work efficiently in such network situations as neither of the two basic protocols works well.

Next comes some comparisons with hybrid routing and Cluster-based Routing. Hybrid routing is another integration of the two basic protocols. Its manner of integration is static and fixed, therefore hybrid routing cannot be adaptive to dynamic network situation. Moreover, Hybrid routing has overlaps of proactive routing zones, and causes more managing overhead of routing information than our system. In Cluster-based Routing, subordinate nodes and cluster-heads work using different routing protocols, and only the cluster-heads have routing information of its clusters. In this sense, our system is similar to the clusterbased routing. However, clusters and their heads must be defined beforehand, it makes network design difficult, and cluster-based routing cannot be adaptive to dynamic network situation. For example, if a cluster-head disappears, a new one must be selected by hand, and the clusters must be re-organized. In our system, if a router-node disappears, its surrounding nodes go back to employ the reactive protocol. Moreover, in the cluster-based routing, every routing must pass through cluster heads, whereas in our system, once the shortest path is found, routing need not pass through any router-node.

We are now at the starting point of this research on adaptive dynamic protocols, and one of the most important issues to address next is collaboration of several router-nodes. We are now investigating, and the below show an outline of the design idea. To collaborate, each router-node must first know other routernodes. Any node who gets to know more than one router-nodes during its routing notifies all the router-nodes. The router nodes get to know the others, and also routes to them in this manner. When a router receives a RREQ packet to an unknown node, It forwards the packet to other routers for inter-cluster routing. We may consider emergence of any super-router-node among router-nodes, similar to IXs in the Internet routing.

\section{Concluding Remarks}

In this research, we designed an adaptive routing in ad-hoc networks. This system can cover the situation where neither of two basic protocols (reactive and proactive ones) work well by maintaining routing information efficiently. It can adapt to the network situation dynamically by changing its routing manner. We implemented a simulator of our design, and showed that it works efficiently.

Further studies include function enhancement. Among them, we are investigating applying the zone concept to our system. In the current design, routing information of the whole network might be accumulated at each router-node in an extreme situation. This unwanted phenomena will be prevented if a routernode has a zone (or a scope) so that it only maintains routes within its zone. 


\section{Acknowledgments}

This research was supported in part by JSPS in Japan under Grants-in-Aid for Scientific Research (B) 17300012, and by MEXT in Japan under Grants-in-Aid for Exploratory Research 17650011.

\section{References}

1. http://www.ietf.org/rfc/rfc3626.txt

2. http://www.ietf.org/rfc/rfc3684.txt

3. http://www.ietf.org/rfc/rfc3561.txt

4. Johnson, D., Maltz, D., Hu, Y., Jetcheva, J.: The Dynamic Source Routing Protocol for Mobile Ad Hoc Networks. IETF MANET Internet Draft, draft-ietf-manet-dsr09.txt (2001)

5. Samar, P., Pearlman, M. R., Hass, Z. J.: Hybrid Routing: The Pursuit of an Adaptable and Scalable Routing Framework for Ad Hoc Networks. in Ad Hoc Wireless Networking (Cheng, X. et al. eds.) Kluwer (2003) 529-560

6. Hass, J. Z., Pearlman, M. R., Sammar, P.: The Zone Routing Protocol (ZRP) for Ad Hoc Networks. IETF MANET Internet Draft, draft-ietf-manet-zone-zrp-03.txt (2002)

7. Jiang, M., Li, J., Tay, Y. C.: Cluster Based Routing Protocol (CBRP) Functional Specification. IETF MANET Internet Draft, draft-ietf-manet-cbrp-spec00.txt (1998).

8. Niegverg, T., Dulman, S., Havinga, P., Hoesel, L. v., Wu, J.: Collaborative Algorithms for Communication in Wireless Sensor Networks. in Ambient Intelligence: Impact on Embedded System Design (Baston, T., et al. eds.) Kluwer (2003) 271-294

9. Wetherall, D.: Active Network Vision and Reality. Proc. 17th ACM Symp. on Operating System Principles (1999) 